\title{
Autoeficacia en salud oral: Concepto, evaluación y tratamiento
}

\section{Self-efficacy in oral health: Concept, assessment and treatment}

\author{
Correa Ramírez A*, Ríos Erazo M*, Herrera Ronda A*, Bustos Reydet C*, \\ Rojas Alcayaga G*
}

\section{RESUMEN}

La autoeficacia es un factor que permite predecir las conductas que un paciente realizará para obtener un adecuado nivel de salud. En salud oral, esto resulta ser fundamental ya que los comportamientos que realiza el paciente determinan la adherencia a las indicaciones que el odontólogo le entrega. El presente artículo aborda la definición de la autoeficacia en general, y en salud oral en particular, además de revisar cuales son los instrumentos más utilizados para medir esta dimensión dentro del contexto odontológico. Finalmente, se revisa un modelo de intervención de la autoeficacia en salud oral que ha mostrado una adecuada efectividad.

Palabras clave: Autoeficacia, salud oral, ciencias del comportamiento.

\section{SUMMARY}

Self-efficacy is a factor that allows predicting patients' behaviours to gain a suitable health level. In oral health this is fundamental, because patients' behaviours determine the adherence to indications provided by the dentist. The present article addresses the definition of self-efficacy in general, in oral health in particular, and a review of the most common instruments to measure this dimension inside the dental context. Finally, it presents a review of an intervention model of self-efficacy in oral health that has shown appropriate effectiveness.

Key words: Self-efficacy, oral health, behavioral sciences.

Fecha de recepción: 30 de enero de 2015.

Aceptado para publicación: 6 de abril de 2015.

* Área de Ciencias del Comportamiento. Instituto de Investigación en Ciencias Odontológicas. Facultad de Odontología. Universidad de Chile.

Correa Ramírez A, Ríos Erazo M, Herrera Ronda A, Bustos Reydet C, Rojas Alcayaga G. Autoeficacia en salud oral: Concepto, evaluación y tratamiento Av. Odontoestomatol 2015; 31 (5): 305-311.

\section{INTRODUCCIÓN}

Los fenómenos que afectan a la salud oral no son sólo explicables desde un punto de vista biológico, también involucra otras dimensiones, tales como: aspectos políticos, económicos, ambientales y psicosociales. La comprensión de las variables que modulan las conductas en salud ayuda a entender mejor la naturaleza de las necesidades, motivaciones y prácticas de los pacientes. Uno de los factores que influye en la ejecución de la conducta en salud es la autoeficacia, la que ha sido considerada como un importante predictor de conductas de salud oral $(1,2)$ y un factor relevante para promover conductas en salud o detener conductas de riesgo asociadas a la pérdida de ésta. El conocimiento del modo como 
opera la autoeficacia y sus alcances en la atención clínica son de vital importancia en la implementación de conductas saludables y constituye para el cirujano-dentista una herramienta eficaz para lograr un mejor estado de salud oral en los pacientes.

\section{CONCEPTO DE AUTOEFICACIA}

Albert Bandura, psicólogo estadounidense, fue quien acuñó el concepto de autoeficacia y la definió como el conjunto de creencias en las propias capacidades para organizar y ejecutar los cursos de acción requeridos para producir determinados logros o resultados (3).

Bajo esta perspectiva, la autoeficacia juega un rol central en la motivación, ya que se relaciona directamente con la confianza que tiene una persona de contar con los recursos personales que le permiten lograr un resultado deseado. Así, las personas con alta autoeficacia buscan desempeñar tareas más desafiantes, colocándose metas y objetivos más altos, además de invertir más esfuerzo en lograr el objetivo que se establecen, siendo más persistentes, y manteniendo un mayor compromiso con sus metas, versus las personas que tienen menor autoeficacia (3). Los juicios que la persona hace acerca de su autoeficacia serían específicos a las tareas y a las situaciones en que se involucra. Esto significa que la autoeficacia no es un atributo general al accionar de un sujeto, sino depende de los dos factores nombrados previamente, tipo de tarea y situación particular.

Bandura define cuatro fuentes de las cuales se derivan los pensamientos de autoeficacia. La primera son las experiencias de éxito, que serían el resultado de las tareas realizadas con resultados satisfactorios para la persona, los cuales serían la fuente de mayor influencia en la autoeficacia. Las experiencias vicarias serían la segunda fuente y son, básicamente, los efectos producidos al observar las acciones de los demás. La tercera fuente sería la llamada persuasión social, que implica la exposición y recepción a los juicios verbales y comentarios de otro y, finalmente, los estados emocionales y fisiológicos como la ansiedad, el estrés, la excitación y la fatiga serían la cuarta fuente, ya que proporciona importante infor- mación e influencia sobre la creencia de la propia eficacia (3).

En salud, muchos de los tratamientos involucran la adopción por parte del paciente de conductas saludables, las cuales deben realizarse de manera sistemática. En la gran mayoría de estas ocasiones el tratante no se encuentra al lado del paciente chequeando si efectivamente lo realiza y cuán bien realiza estas conductas. De esta forma, la autoeficacia resulta relevante, ya que le permite al profesional de la salud predecir si el paciente realizará dichos comportamientos saludables. Así, en el contexto sanitario, la autoeficacia correspondería a la creencia de un paciente de desarrollar e implementar ciertas conductas favorables para su salud. Diversas investigaciones revelan que altos niveles de autoeficacia tienen consecuencias beneficiosas para el funcionamiento del individuo y su bienestar general (3).

La autoeficacia en salud ha sido aplicada a conductas tan diversas como, por ejemplo, el manejo de enfermedades cardíacas (4), osteoporosis (5), artritis (6), adherencia a la medicación (7), el realizar ejercicio (8), el bajar de peso (9), así como también para los resultados de la rehabilitación (10). En relación a las conductas preventivas en salud, los individuos con alta autoeficacia percibida tienen más probabilidad de iniciar cuidados preventivos, buscar tratamientos tempranos y ser más optimistas sobre la eficacia de estos (11). Además, los estudios indican consistentemente que los individuos con alta autoeficacia percibida también tienen más probabilidad de evaluar su salud como mejor, presentar menos indicadores depresivos y recuperarse mejor y más rápidamente de las enfermedades que las personas con baja autoeficacia percibida (11).

\section{AUTOEFICACIA EN SALUD ORAL}

Dentro de la salud bucal, la autoeficacia también cumple un rol preponderante. Esta es definida como las creencias en las propias capacidades personales para organizar, y ejecutar rutinariamente los cursos de acción necesarios para mantener los tejidos orales en buenas condiciones. La falta de una rutina de autocuidado en el hogar es una situación frecuente en la mayoría de los adultos (12), lo que dificulta una 
adecuada salud oral por la no adopción de conductas favorables a ésta, lo que muestra la relevancia del concepto de autoeficacia.

Bajo esa perspectiva, existen múltiples investigaciones que dan cuenta de la relación de autoeficacia y ciertas conductas favorables para la salud oral, como el cepillado dental y el uso de seda dental frecuente (13-15). Otras conductas favorables en salud oral son el tener buenos hábitos dietéticos, la adherencia a medicamentos prescritos, o las visitas al dentista, tanto para un tratamiento como para controles de rutina. Se ha demostrado que personas con altos niveles de autoeficacia para cepillado, para hábitos dietéticos y para visitas al dentista, se correlacionan con niveles más altos de salud oral y menores índices de sangrado al sondaje que aquellos que tienen bajos niveles de autoeficacia (16). ¿Por qué estas conductas son importantes? Porque se sabe que el control mecánico de la placa bacteriana previene la severidad de la inflamación gingival, y está demostrado que la disminución en el consumo de bebidas azucaradas, es un indicador fuerte de caries dental, incluso mayor que el consumo de alimentos "snack" (17). La autoeficacia es un buen predictor de la capacidad del paciente para completar un tratamiento odontológico (18), situación vinculada a una mejor condición de salud oral dado que se ha demostrado la efectividad del cuidado profesional en el tratamiento y prevención de enfermedades periodontales (19).

\section{LA AUTOEFICACIA Y SU EVALUACIÓN}

Entendiendo la importancia de la autoeficacia como un adecuado predictor de conductas, es que resulta fundamental conocer sus mecanismos de evaluación. A nivel global, la Escala de Autoeficacia General de Schwarzer y Jerusalem es la más utilizada (10). Este instrumento mide autoeficacia desde un punto de vista psicológico global, y se compone de 10 ítems, cuyo formato de respuesta está enumerado del 1 al 4, de menor a mayor nivel de acuerdo con la afirmación de cada ítem. Su confiabilidad corresponde a un Alfa de Cronbach que va en un rango de 0,76 a 0,90 , mostrando un promedio 0,80 en una muestra de 26 países, presentando además una adecuada validez. En Chile, esta escala fue validada por Cid,
Orellana y Barriga, obteniendo una adecuada validez y confiabilidad, con un coeficiente alfa de Cronbach de 0,84 (21).

En relación a hábitos saludables, se han construido distintos instrumentos para medir autoeficacia. Ejemplos de aquellos son la escala de autoeficacia en pacientes cardíacos (22), o la escala de autoeficacia que mide la creencia de los participantes sobre cuán capaces son de realizar cantidades crecientes de ejercicio en una intensidad moderada a severa (23). También existe una escala para medir autoeficacia en relación a la adherencia al tratamiento farmacológico (24), y la escala de autoeficacia en estrategias de reducción del consumo la marihuana (25).

La autoeficacia en salud oral resulta ser un área de investigación que comenzó su desarrollo en forma reciente, por lo que los estudios, si bien existen, son limitados en comparación con otras áreas de la salud. La limitación se hace más evidente cuando uno observa investigaciones de habla hispana. Dentro de las investigaciones realizadas en relación a la creación de instrumentos, existen dos escalas a destacar: el primero es el realizado por Kakudate y Morita, quienes desarrollaron una escala de autoeficacia para el autocuidado para pacientes con periodontitis crónica (26). Esta consiste en 15 ítems divididos en 3 subescalas: La autoeficacia para consultas con el dentista, que son 5 ítems que buscan evaluar factores relacionados a la adherencia y controles regulares con el dentista; la autoeficacia para el cepillado dental, formada por 5 ítems que buscan evaluar el cuidado y la meticulosidad con que el paciente se lava sus dientes; y finalmente la autoeficacia para hábitos dietéticos, que son 5 ítems relacionados con la adopción de una alimentación equilibrada y hábitos de ingesta de líquidos. Todas las respuestas reciben un puntaje según una escala Likert de 5 niveles, en un rango que va desde un puntaje de " 1 " (no seguro) a "5" (completamente seguro). La sumatoria de estos 15 ítems entrega el total de puntos de la escala. La confiabilidad del instrumento fue evaluada a través de técnicas convencionales, obteniendo un alpha de Cronbach de 0,86 y una estabilidad testretest de 0,73 . También se demostró una adecuada validez de constructo por medio de un estudio que encontró que los pacientes periodontales con una terapia exitosa de mantención presentaban resulta- 
dos altos en la escala versus los pacientes que no recibieron tratamiento periodontal (26).

El segundo instrumento es el cuestionario de autoeficacia dental, diseñado por Syrjälä, quien modificó el cuestionario original creado por Lawrence en el año 1985. La escala consiste en preguntas sobre la autoeficacia relacionada con el cepillado, la limpieza interdental y las visitas al dentista, todas con 4 posibles respuestas: completamente seguro que sí, bastante seguro que sí, bastante seguro que no y completamente seguro que no. Cabe mencionar que esta se encuentra en inglés porque aún no ha sido adaptada al idioma español. La escala presentó una adecuada confiabilidad, con alfas de Cronbach superiores a 0,90 en cada una de sus subdimensiones (27).

\section{MODIFICANDO LA AUTOEFICACIA EN SALUD ORAL}

Entendiendo la relevancia que tiene la autoeficacia en la salud oral de los pacientes, resulta fundamental conocer cuáles serían los mecanismos que permitirían optimizar esta variable. Farquhar $(28,29)$ establece un modelo genérico de facilitación del cambio de la autoeficacia. Bajo este parámetro, fue Kakudate quien modificó dicho modelo para su intervención específica en el área de la odontología (Figura 1). Esta intervención ha mostrado una mayor efectividad en la mejora de la autoeficacia y en el desarrollo de cambios conductuales en higiene oral en pacientes con periodontitis crónica, que la sola instrucción de higiene oral.

A continuación veremos cada uno de los 6 pasos (30):

El primer paso se conoce como identificar el problema. Acá, el dentista debe clarificar las creencias, el conocimiento y las barreras que tendría el paciente para realizar un adecuado autocuidado periodontal. Se recomienda que esta información se obtenga por medio de una interacción cara a cara, teniendo como guía algunas preguntas como: ¿Cuántas veces y cuánto tiempo dura su cepillado dental?, cqué sabes sobre el autocuidado en salud oral?, cha tratado de cambiar tu conducta en el pasado? y cंcuáles son las mayores barreras que existen para que se genere un cambio? Este paso permite establecer e identificar en conjunto con el paciente sus comportamientos dañinos y cuáles son los cambios conductuales que se deben implementar (30).

El segundo paso es el generar confianza $y$ optimismo. Muchos pacientes tienen la firme convicción de que su cambio de conducta no va a ser posible. Por lo tanto, este paso pretende aumentar la confianza y el compromiso en el paciente. ¿Cómo? A través de la entrevista que se realiza inicialmente, incorporando intervenciones que permitan al paciente superar las barreras para el cambio de conducta. Un ejemplo de intervención podría ser el referirse a un paciente, que en una situación similar pudo realizar los cambios necesarios. Para promover la motivación y compromiso, se puede firmar un contrato donde tanto dentista como paciente se comprometan a trabajar en un cambio de conducta determinado (30).

El tercer paso sería el aumentar la conciencia de la conducta. Este paso busca aumentar la conciencia de la conducta del paciente, por medio del automonitoreo. Esto se logra solicitando que registre en una bitácora su frecuencia de cepillado dental y limpieza interdental hasta la siguiente consulta, además de registrar sus sentimientos/emociones o sensaciones cuando se lavaba los dientes. Esta bitácora permite determinar los generadores internos y externos de comportamiento, además de identificar más claramente las barreras para el cambio conductual en relación al cuidado en salud oral, ya que en muchas ocasiones el paciente no tiene clara dichas barreras (factor consultado en el paso uno), por lo que esta bitácora pone en evidencia las barreras no conscientes (30).

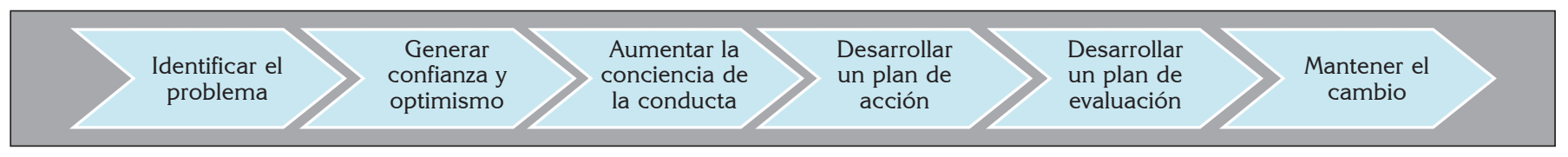

Fig. 1. Modelo de intervención de autoeficacia en salud oral de Kakudate. 
El cuarto paso sería el desarrollar e implementar un plan de acción. Esto se puede realizar por medio de la descripción de la bitácora solicitada para el paciente que permitiría identificar los cuidados en salud oral que presenta, donde sería posible generar un plan de acción basado en la gradualidad. Este plan de acción debe tener ciertas características particulares, como ser concreto, realista y sobretodo alcanzable. Por ejemplo, un plan de acción adecuado podría incluir el "lavarse los dientes dos veces por día", "cepillarse por más de tres minutos" y "usar seda dental una vez al día en la noche". Si el paciente logra estas conductas, puede recompensarse por medio de incentivos pequeños, como un tratamiento de belleza, o ver una película, por ejemplo (30).

El quinto paso es el desarrollar un plan de evaluación. Este paso debe realizarse sí o sí, independiente si el paciente tuvo éxito o no en el logro del plan de acción. Si logra éxito, este debe ser reforzado positivamente por el dentista, lo que generará por consiguiente una mayor frecuencia de ejecución de esta conducta. Por el contrario, si no logra el éxito, la idea es reformular el plan ejecutado, ajustando objetivos o chequeando obstáculos que surgieron (30).

El sexto y último paso sería mantener el cambio y prevenir las recaídas. Es conocido que la adquisición de nuevas conductas puede ser complejo a largo plazo, ya que existen muchos imponderables que pueden alterar esa regularidad de la conducta, como largas horas de trabajo, eventos sociales, por nombrar algunos. Es bajo esta perspectiva que el dentista ayude y refuerce constantemente al paciente para que cuide sus nuevos comportamientos adquiridos. Una forma de reforzar sería la técnica utilizada en el paso cuatro, utilizando incentivos luego de ejecutada la conducta, los cuales pueden ser aplicados durante un largo tiempo, todo con el objetivo de mantener dichas conductas (30).

\section{CONCLUSIONES}

La autoeficacia es considerada como un importante predictor de conductas en salud, además de ser un elemento central a la hora de implementar cambios significativos en el bienestar de las personas. Su uso exitoso en ámbitos sanitarios tan diversos, como el cardiovascular, nutricional o mental, fomenta su abordaje desde el ámbito de la salud oral, donde las conductas de autocuidado que implementa el paciente son centrales a la hora de obtener indicadores orales óptimos.

Si bien existen una gran cantidad de instrumentos que permiten evaluar la autoeficacia en distintos ámbitos de la salud, en odontología los estudios son más bien limitados. Por esto, resulta recomendable la realización de investigaciones que busquen crear escalas para esta disciplina, para así lograr identificar con la mayor confiabilidad y validez posible la autoeficacia en salud oral del paciente.

Por último, lograr una intervención a nivel de la autoeficacia en salud oral, basándose en criterios empíricos, permitiría una mejor adherencia al tratamiento dental, lo que consecuentemente generaría una mejoría en la salud oral para el paciente y un menor gasto público en salud oral, debido a que son intervenciones de bajo costo y altos beneficios.

\section{BIBLIOGRAFÍA}

1. Schwarzer R, Renner B. Social-Cognitive Predictors of Health Behavior: Action Self-Efficacy and Coping Self-Efficacy. Health Psychol 2000;19 (5):487-95.

2. Strecher JV, McEvoy B, Becker MH, Rosenstock IM. The Role of Self-Efficacy in Achieving Health Behavior Change. Health Educ Behav 1986;1 (13):73-92.

3. Bandura A. Self efficacy: Toward a Unifying Theory of Behavioral Change. Psychol Rev. 1977; 84:191-215.

4. Sullivan MD. Andrea Z, Russo J, Katon WJ. SelfEfficacy and Self-Reported Functional Status in Coronary Heart Disease: A Six-Month Prospective Study. Psychosomatic Med 1998;60(4):473-8.

5. Horan ML, Kim KK, Gendler P, Froman RD, Patel MD. Development and evaluation of the Osteoporosis Self-Efficacy Scale. Res Nurs Health 1998; 21(5):395-403. 
6. Lorig K, Chastain RL, Ung E, Shoor S, Holman HR. Development and evaluation of a scale to measure perceived self-efficacy in people with arthritis. Arthritis Rheum 1989;32:37-44.

7. Fernández S, Chaplin W, Schoenthaler A, Ogedegbe G. Revision and Validation of the Medication Adherence Self-Efficacy Scale (MASES) in hypertensive African Americans. J Behav Med 2008;31(6):453-62.

8. Resnick B, Jenkins LS. Testing the reliability and validity of the Self-Efficacy for Exercise scale. Nurs Res 2000;49(3):154-9.

9. Resnik B, SI Zimmerman, D Orwig, A-L Furstenberg, J Magaziner. Outcome Expectations for Exercise Scale: Utility and psychometrics. J Gerontol B Psychol Sci Soc Sci 2000;55(6): S352-6.

10. Waldrop D, Lightsey OR, Ethington CA, Woemmel CA, Coke AL. Self-efficacy, optimism, health competence and recovery from orthopedic surgery. J Couns Psychol 2001;48:233-8.

11. Oliverí C, Urra E. Autoeficacia y conductas de salud. Cienc. Enferm 2007;13(1):9-15.

12. Ciancio, S. Improving oral health: current considerations. Journal of Clinical Periodontology 2003;30:4-6,

13. Schwarzer R, Antoniuk A, Gholami M. A brief intervention changing oral self-care, self-efficacy, and self-monitoring. $\mathrm{Br} \mathrm{J}$ Health Psychol 2015;20(1):56-67.

14. Buglar M, White K, Robinson N. The role of self efficacy in dental patient's brushing and flossing: testing an extended Health Belief Model. Patient Educ Couns. 2010;78(2):269-72.

15. Blinkhorn A. Influencing patients' oral hygiene behaviours. Evid Based Dent 2010;11(2):41.

16. Mizutani S, Ekuni D, Furuta M, Tomofuji T, Irie K, Azuma T, Kojima A, Nagase J, Iwasaki Y, Morita $M$. Effects of self-efficacy on oral health behaviours and gingival health in university students aged 18- or 19-years-old. J Clin Periodontol 2012;39:844-9.

17. Jain P, Gary JJ. Which is a stronger indicator of dental caries: oral hygiene, food, or beverage? A clinical study. Gen Dent 2014; 62(3):63-8.

18. Kakudate N, Morita M, Kawanami M. Oral health care specific self-efficacy assessment predicts patient completion of periodontal treatment: a pilot cohort study. J Periodontol 2008;79:10417.

19. Axelsson P, Nyström B, Lindhe J. The long-term effect of a plaque control program on tooth mortality, caries and periodontal disease in adults. Results after 30 years of maintenance. J Clin Periodontol 2004;31:749-57.

20. Schwarzer, R., E Jerusalem, M.. Generalized SelfEfficacy scale. En: Weinman J, Wright S, Johnston M. Measures in health psychology: A user's portfolio. Causal and control beliefs. NferNelson: Windsor; 1995.

21. Cid P, Orellana A, Barriga O. Validación de la escala de autoeficacia general en Chile. Rev. Méd. Chile 2010;138(5):551-7.

22. Sullivan M, LaCroix A, Russo J, Katon W. SelfEfficacy and Self-Reported Functional Status in Coronary Heart Disease: A Six-Month Prospective Study. Psychosomatic Med 1998;60:473-8.

23. McAuley E, Talbot H-M, \& Martinez S. Manipulating self-efficacy in the exercise environment in women: Influences on affective responses. Health Psychology 1999; 18(3): 28894.

24. Fernandez S, Chaplin W, Schoenthaler AM, Ogedegbe G. Revision and validation of the medication adherence self-efficacy scale (MASES) in hypertensive African Americans. J Behav Med. 2008;31:453-62.

25. Davis AK et al. Psychometric evaluation of the Marijuana Reduction Strategies Self-Efficacy 
Scale with young recreational marijuana users. Addict Behav. 2014;39(12):1750-4.

26. Kakudate N, Morita M, Fujisawa M, Nagayama M, \& Kawanami M. Development of the SelfEfficacy Scale for Self-care (SESS) among periodontal disease patients (in Japanese). J Jpn Soc Periodontol 2007;49:285-95.

27. Syrjälä, A-M H, Kneckt MC, Knuuttila MLE. Dental self-efficacy as a determinant to oral health behaviour, oral hygiene and $\mathrm{HbA} 1_{c}$ level among diabetic patients. Journal of Clinical Periodontology 1999;9(26):616-21.

28. Albright CL, Farquhar JW Principles of behavioral change. En: Greene HM. Introduction to Clinical Medicine. Decker: London; 1992.
29. Farquhar JW. The American way of life need not be hazardous to your Health. Da Capo: New York City; 1987.

30. Kakudate N, Morita M, Sugai M, Kawanami M. Systematic Cognitive Behavioral Approach for Oral Hygene Instruction: A Short-Term Study 2009;2(74):191-6.

\section{CORRESPONDENCIA}

Matías Ríos Erazo.

Sergio Livingstone P. 943, Independencia

Santiago, Chile.

Correo electrónico: matiasrios@gmail.com 